\title{
Review of Ad Hoc Networks scenarios and challenges in years 2015-2019
}

\author{
Amna Saad \\ Universiti Kuala Lumpur, \\ Malaysia Institute of Information Technology \\ 1016, Jalan Sultan Ismail, Bandar Wawasan, 50250 Kuala Lumpur, \\ Wilayah Persekutuan Kuala Lumpur, Malaysia \\ amna@unikl.edu.my
}

\author{
Husna Osman \\ Universiti Kuala Lumpur, \\ Malaysia Institute of Information Technology \\ 1016, Jalan Sultan Ismail, Bandar Wawasan, 50250 Kuala Lumpur, \\ Wilayah Persekutuan Kuala Lumpur, Malaysia \\ husna@unikl.edu.my

\section{Mufind Mukaz Ebedon} \\ Universiti Kuala Lumpur, \\ Malaysia Institute of Information Technology \\ 1016, Jalan Sultan Ismail, Bandar Wawasan, 50250 Kuala Lumpur, \\ Wilayah Persekutuan Kuala Lumpur, Malaysia \\ mufind.mukaz@s.unikl.edu.my
}

\begin{abstract}
A Mobile Ad-hoc Network (MANET) protocol performance analysis depends on the type of simulation tools, mobility models, and metrics used. These parameters' choice is crucial to researchers because it may produce an inaccurate result if it is not well chosen. The challenges researcher is facing are on the choice of these four parameters. Our survey shows an inclination to used Ad-hoc On-Demand Distance Vector routing (AODV) for performance comparison and enhancement of it by the researcher. Network simulation 2 (NS2) was the most selected tool, but we observe a decline in its utilization in recent years. Random Waypoint Mobility model (RWPM) was the most used mobility model. We have found a high percentage of the published article did not mention the mobility models use; this will make the result difficult for performance comparison with other works. Packet Delivery Ratio (PDR), End to End Delay (E2ED) were the most used metrics. Some authors have self-developed their simulation tools; the authors have also used new metrics and protocols to get a particular result based on their research objective. However, some criteria of choosing a protocol, metrics, mobility model, and simulation tool were not described, decreasing the credibility of their papers' results. Improvement needs to be done in the Ad-hoc network in terms of benchmark, acceptable scenario parameters. This survey will give the best practice to be used and some recommendations to the Ad-hoc network community.
\end{abstract}

Keywords: MANET, Routing protocols, Mobility Model, Metric, Simulation tool, Performance analysis

\section{INTRODUCTION}

MANET can be implemented without any centralized administration. Mobile nodes in the Ad-hoc network form a network on the go; each mobile node can be a forwarding node and sending node simultaneously.

Ad-hoc networks can be classified into Mobile Adhoc Networks (MANET), Vehicular Ad-hoc Network (VANET), Wireless Sensor Network (WSN), and Flying Ad-hoc Network (FANET). Ad-hoc networks need a protocol that adapts to these parameters: topology change, updating of a new route path, and energyefficient of the node.

Designing a protocol that will include these criteria and give a better result in a different scenario is difficult to achieve. Various proto-cols have been proposed in the literature, such as Ad- hoc On-demand Distance Vector (AODV) [1], Dynamic Source Routing (DSR) [2], Dynamic Manet On-demand (DYMO) [3], Ad-hoc Ondemand Multipath Distance Vector (AOMDV) [4], Multicast Ad-hoc On-demand Distance Vector (MAODV) [5], Destination Sequenced Distance Vector (DSDV) [6], 
Optimized Link State Routing (OLSR) [7], Zone Routing Protocol (ZRP) [8] and Low-energy Adaptive Clustering Hierarchy (LEACH) [9].

The mobile nodes in ad-hoc networks move arbitrarily, and the topology of the network change frequently. The mobile node's arbitrary movement makes it hard for a researcher to find the mobility models close to the reality (human movement) for implementation in the simulation tools.

Here are some well-known simulation tools in Adhoc network : Network simulator 2 (NS2) [10], Network Simulator 3 (NS3) [11], Objective Modular Network Testbed in C++ (OMNet++) [12], Optimized Network Engineering Tool (OPNET) [13], Global Mobile Information System Simulator (GloMoSim) [14], Matrix Laboratory (MATLAB) [15], Castalia [16] and EXATA Cyber [17].

The mobility model in Ad-Hoc Networks shows how the mobile nodes move in the network. There are different mobility models based on their movement patterns, such as Random Way Point Mobility Model (RWPM), Random Walk Mobility Models (RWM), Reference Point Group Mobility Models (RPGM), Gauss-Markov Mobility Models (GMM) [18].

Open Simulation of Urban Mobility (SUMO) combines with a Mobility generator for Wireless Networks (MOVE) [19, 20], and MOBISIM [21, 22]. Metrics play a significant role in data analysis; some well-known metrics are Packet Delivery Ratio (PDR), Average Overhead, Throughput, End-to-End Delay E2ED, Energy consumption, and Jitter.

The review analyses 169 papers retrieved from 5 Scopus journals selected based on their focus on mobile ad-hoc networks and other criteria from 2015 to 2019. The result shows that $45.6 \%$ of the researchers used Adhoc On-Demand Distance Vector routing (AODV) as a protocol for their performance comparison, 37.9\% used the Random Waypoint Mobility Model (RWPM) in their simulation scenarios. 52.1\% used Packet Delivery Ratio (PDR) as their metrics, and $60.9 \%$ used Network Simulation 2 (NS2) as the simulation tool. We have observed that NS2 utilization is declining in recent years because it does not support new technology (loT, 5G). Some papers did not mention the mobility model used, which can create difficulty for the researcher to compare with other works. The researcher will face some challenges when selecting the simulation tools, the protocol for comparison or enhancement, choice of mobility model, and metrics to use for a better view of the networks. Our review shows what parameters have been used as a reference for future researchers. We have proposed some best practices and some recommendations to the ad hoc network community for improvement.

The rest of this review is organized as follows: Section 2 reviews existing literature, Section 3 gives detail of the review methodology, the result is provided in Section 4. Section 5 discusses the result and recommendation. Finally, Section 6 contains the conclusion.

\section{LITERATURE REVIEW}

Yoon et al. [23] studied the random waypoint and found out that it is not a good mobility model to use because unreliable results can be obtained using this model. They observe that if the simulation time increases, some metrics show a drop in performance. They proposed a new modified Random mobility model.

Kurkowski et al. [24] surveyed proceeding paper in Association for Computing Machinery (ACM) on MANET from 2000-2005. They extracted simulation parameters from those surveyed papers. They found out that NS2 was the most simulation tool used by authors and RWPM as a mobility model. Some missing parameters were observed, and the unavailability of code was missing for self-developed simulation tools.

Hiranandani et al. [25] conducted a review of published papers in the ACM conference between 2006 to 2010. They found out that missing parameters are still observed. Default parameters for simulators have been used by research, which raises a question on the result's credibility. They observed that since the study in [24], the current mobile ad hoc simulation practices are still not progressing. Still, some custom simulators were more used compared to existing simulator tools.

Naicken et al. [26] surveyed 280 papers in the peerto-peer network to see what simulation tools were used. They found out that custom simulators surpass the well-known simulators in terms of usage.

Kurkowski et al. [27] proposed a simulation standard by using two metrics for characterization of the simulation scenarios, such as the average shortest path hop count and the average amount of network partitioning. Enhancement of Kurkowski works was done in [28], which adds a new metric average neighbour count.

Andel et al. [29] focused on the credibility of manet simulation tools. By analyzing multiple review papers published, they found out that most authors do not specify their simulator's version, and missing simulation parameters can be observed. They proposed some solutions to improve the credibility of the simulation tools.

Ahmad et al. [30] proposed a comprehensive comparison of AODV, DSDV, and ZRP protocol by reviewing some related works in terms of routing used, simulator tool, metrics, network type, and qualitative analysis was done.

Sanchez et al. [31] surveyed Unmanned aerial and aquatic vehicles, and the focus was on their communication, application, and tools for the evaluation.

Garcia et al. [32] proposed a methodology to help the researcher conduct good simulation practice in their scenarios in VANET. Different simulation scenarios were proposed in NS2 to determine an excellent method to use.

Other research focuses on the performance comparison of different simulation tools [33-36]. 
To the best of our knowledge, no review paper has covered the literature on performance evaluation or analyses of articles in Ad-Hoc networks from 2015 to 2019 in MANET, WSN, FANET, and VANET based on different parameters. Therefore, this study aims is to conduct a literature review on the performance evaluation to:

a) Identify the mobility model, metrics, simulation tools, and routing protocols used in MANET, VANET, WSN, and FANET with a synthesis of empirical evidence

b) To analyze the result and present our finding

\section{REVIEW METHODS}

This review will show the process of formulating the research questions, which include the search processes that represent the keyword and the Inclusion-Exclusion criteria for selecting the papers. The following are the formulating research questions for our review:

a) RQ1: Which of the simulation tools, mobility models, metrics, and routing protocol are the most used in the performance analysis of MANET, VANET, WSN, and FANET?

b) RQ2: What are the lessons learned and best practices in the performance analysis of ad hoc networks?

\subsection{SEARCH PROCESSES}

The literature has been searched from selected Scopus journals, which are focusing on ad-hoc networks IEEE Access (IEEE), IEEE Transactions on communication (IEEE), Wireless Networks (Springer), Wireless Personal Communication (Springer), and Ad-hoc networks (Elsevier). The criteria for selecting these journals were based on the higher number of published articles in ad hoc networks compared to other journals.

The keyword search in the article and the abstract was: "performance comparison" and "mobility model" and "simulation" and "ad-hoc networks" or secondary keyword "performance evaluation" and "mobility model" and "routing protocols" and "ad-hoc networks" in those five journals ranging from 2015 to 2019. Retrieving paper after using the keyword was analyzed first by screening the title to see if it is relevant to our objective; if yes abstract was read to double confirm if the paper can be selected or not. A full reading of the selected paper was done to see if the paper contains these parameters (metrics, simulation tools, protocol, mobility models). For protocol, we included the article, which details the protocol used for the implementation of the scheme or algorithm, and those who compared new protocol with existing protocols. We need to clarify that the compulsory parameters for inclusion were the Simulation tools, metrics, and protocols. The articles that do not show the mobility model but have all the other parameters were selected in our review.

\subsection{INCLUSION-EXCLUSION CRITERIA}

The selection of the articles was conducted based on the inclusion and exclusion criteria. The articles which fulfil the criteria in Table 1 were selected, and those that do not were excluded in Table 2.

Table 1. Inclusion criteria for the selection of articles

\begin{tabular}{l} 
Inclusion criteria \\
\hline Publication date 2015-2019 \\
\hline English
\end{tabular}

Any geographical location

The articles published in IEEE Access, IEEE Transactions on communication, Wireless Networks, Wireless Personal communication, and Ad-hoc networks.

Performance comparison, routing protocols, and mobility model in ad-hoc networks must be the primary topic or secondary topic of the publication

The article should report the simulation parameters table or a simulation parameter description and should include the protocol, simulation tools

Table 2. Exclusion criteria for the selection of articles

Exclusion Criteria
Published pre-2015
Non-English
Proceedings and peer-reviewed papers, and articles published in
other journals, patent.

The proposal, lectures notes, A summary of conference Keynote, Dissertation/Thesis, Doctoral Workshop, and tutorial

Articles did not present the simulation parameters table or a simulation parameter description such as (protocol, metrics, simulation tools)

An article which presents algorithm and schemes without comparing it to the new protocol or without describing in what protocol it was implemented were excluded

\section{RESULTS}

The flow diagram in Figure 1 shows the articles' selection process for the review. The following number of articles from 5 Scopus journals have been retrieved from 3 databases (IEEE, Springer, Elsevier): From IEEE two journals; IEEE Access (429), IEEE Transaction on communication (127), from Springer 2 journal; Wireless Networks (306) and Wireless Personal communication (384) and finally from Elsevier Ad-hoc networks (400). A total of 1646 articles were identified. After the title and abstract filtering, 796 articles were excluded because they were not relevant to the topic and did not focus on ad-hoc networks. We have conducted a full article review on the remaining 850 articles, and 672 were excluded due to missing some parameters listed in the Exclusion criteria, as illustrated in Table 2. The remaining 169 were selected for the review. 


\section{DISCUSSION}

A. Which of the simulation tools, mobility models, metrics, and routing protocol are the most used in the performance analysis of MANET, VANET, WSN, and FANET?

To answer RQ1, we have extracted 169 articles with the following information. For a summary result see Figure 4.

\subsection{SIMULATION TOOLS}

Figure 2 shows the network simulator tools used in all the articles we have reviewed from 2015 to 2019 . We can observe an inclination toward free simulator tools than the paid ones. Network simulator 2 (NS2) was the most used with 103 (60.9\%) out of 169 articles, 13 (7.7\%) NS3, 14 (8.3\%) MATLAB, 7 (4.1\%) used OMNET++ and OPNET Modeler, $5(3 \%)$ GloMoSim and Qualnet and 3 (1.8\%) EXATA/Cyber. Figure 3 shows that 70 out of 99 $(77.7 \%)$ in MANET, 15 out of $32(46.8 \%)$ in VANET and 18 out of $37(48.6 \%)$ in WSN. MATLAB and OPNET surpass NS3 in terms of usage in MANET. OMNET++ and NS3 have similar articles used in VANET.

NS2 has shown a high percentage of usage in our review because it is a free license simulator. NS2 has multiple models, many protocols are implemented in it, the source code is available for free, and the documentation can be found on the NS website and other pages [37]. The negative side is that it does not contain new features that can support other research areas like the Internet of Things (IoT), 5G. Another disadvantage is that NS2 code cannot be implemented directly to a real system due to multiple languages ( $\mathrm{TCL}, \mathrm{C}++$ ). Comparing our result with the result in [24] we can observe similarities in terms of utilization of NS2. Our survey shows $60.9 \%$ out of 169 papers and $43.8 \%$ out of 80 for the previous review. The result in [25] shows that a custom simulator was more used, but fewer authors used a custom simulator in our research. This review has observed a decrease in the utilization of NS2 in the current year, and improvement has been seen since the work in [25] that standard simulation is more used than custom simulation tools, which can help researchers to repeat the work.

We can conclude that there are no predefined simulator tools to use in a particular research area, as long as the code's availability is there, and the evaluation methodology is well designed. New simulators are available, which can bring new features and models to the research area. Still, the challenges are the source code's availability, implementation of various models, and documentation.

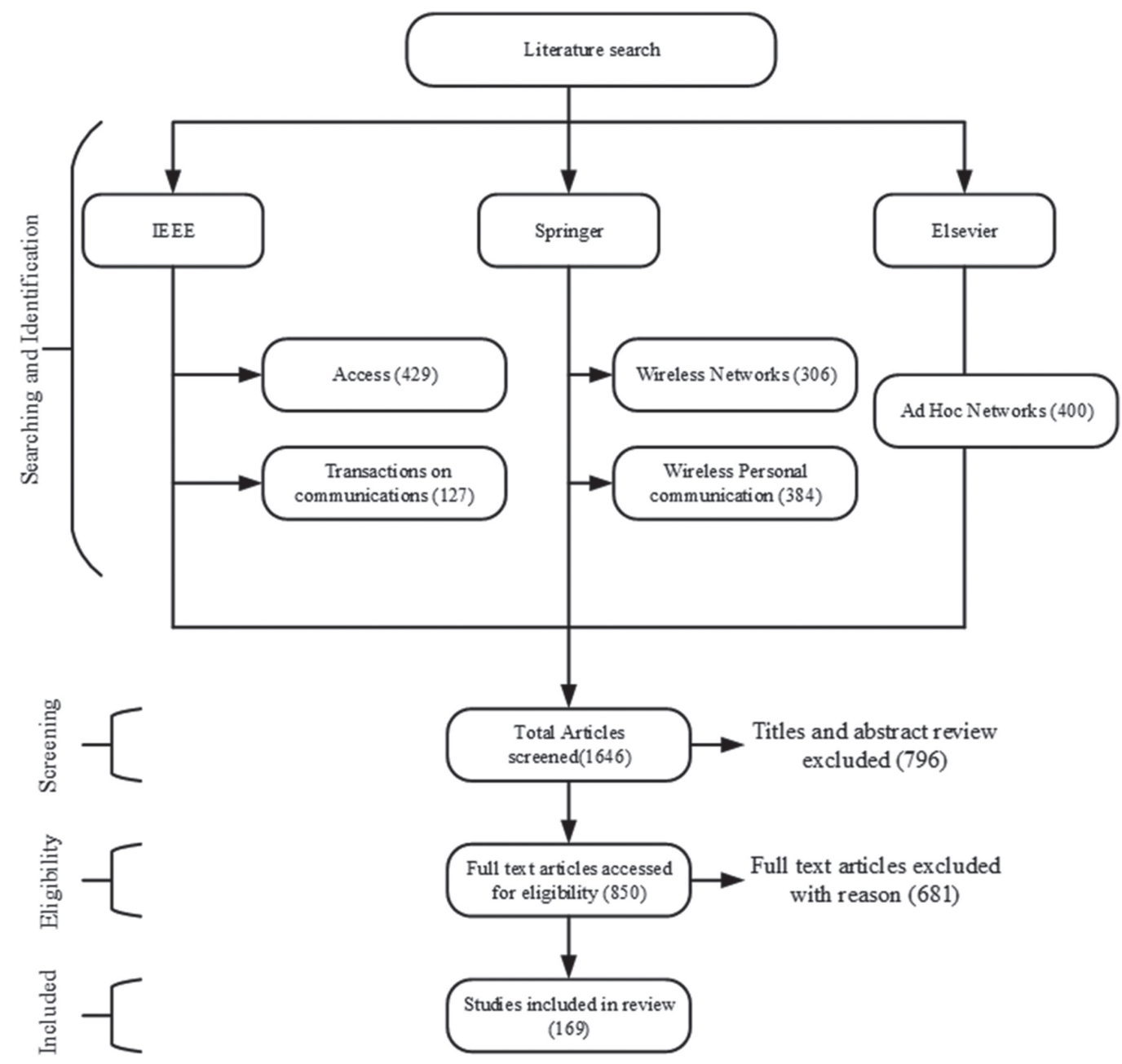

Fig. 1. The flow diagram of the article for the literature review 


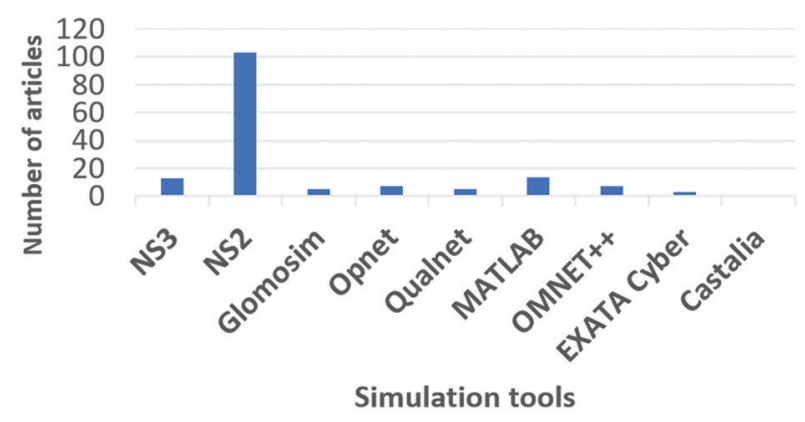

Fig. 2. Simulation tools usage

\subsection{PROTOCOLS}

The studies of protocols are very important before deciding on what protocol to enhance or compare with when designing an evaluation study. The nonavailability of some protocol source code makes it difficult for the researcher to enhance or compare with their new protocol. Figure 5, shows $77(45.6 \%)$ out of 169 articles used AODV, 27 (16\%) DSR, 17 (10.1\%) OLSR, 9 (5.3\%) LEACH, 8 (4.7\%) DSDV, 6 (3.6\%) DYMO, 9 (5.3\%) AOMDV, 5 (3\%) MAODV and 3 (1.8\%)ZRP. Table 3 shows protocols that have a low percentage of utilization. This study's trend indicates that reactive protocol is preferred for performance comparison with the new one. Figure 6 shows the most protocol used in ad-hoc networks, 50 out of 99 (55.5\%) articles used AODV in

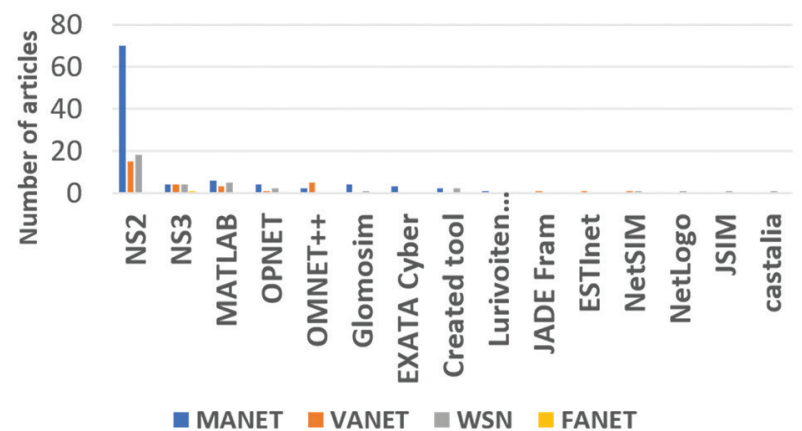

Fig. 3. Simulation tools usage base on MANET, VANET, WSN, FANET

MANET, in VANET 12 out of 32 (37.5\%), WSN 14 out of $37(37.8 \%)$, and 1 out of 1 article in FANET, the next protocol which was most used is DSR. LEACH was only used in WSN 8 out of 37 (21.6\%).

The challenge facing is on what protocol to compare with and in what scenarios. Comparing an existing protocol with a new one helps to view the new one's positive and negative aspects. The question is, in what situation should a protocol be chosen for comparison. Research in [38] compared AODV and ZRP with their proposed protocol GeoZRP. Why not compare it Directly to ZRP or compare it with protocol base on their focus, e.g., security, energy, multipath. Those are the challenges researchers face in the selection of protocol to analyze or enhance.

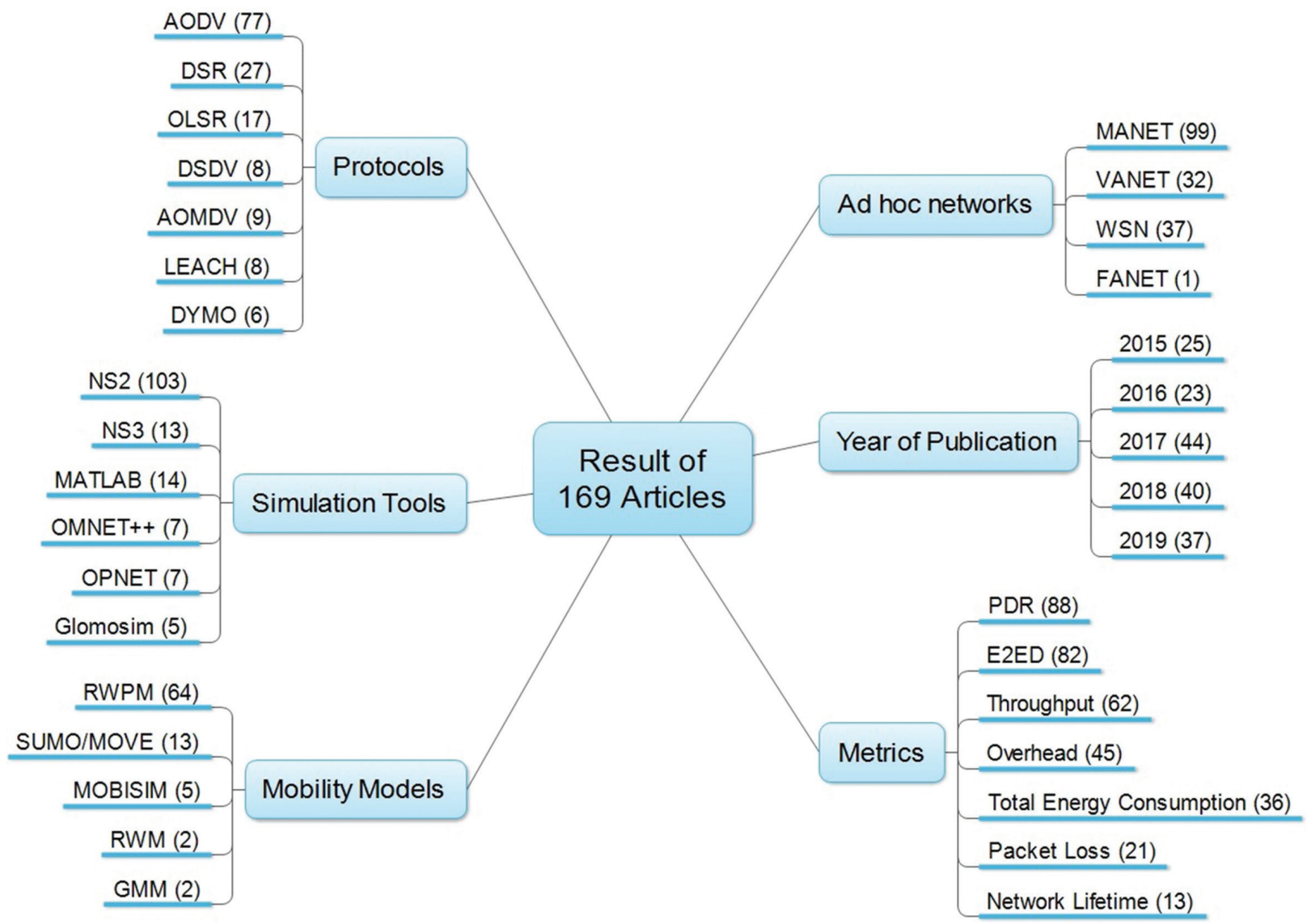

Fig. 4. Summary result of 169 articles selected in the review 
We can conclude that a solution to solve this problem is for research to implement new protocols in well-known simulators tools and make the source code available. It will enable future researchers in ad-hoc networks to have the possibility to modify, reproducing, and confirming their results with the new existing protocol, rather than comparing it with an old protocol because of its availability of the source code.

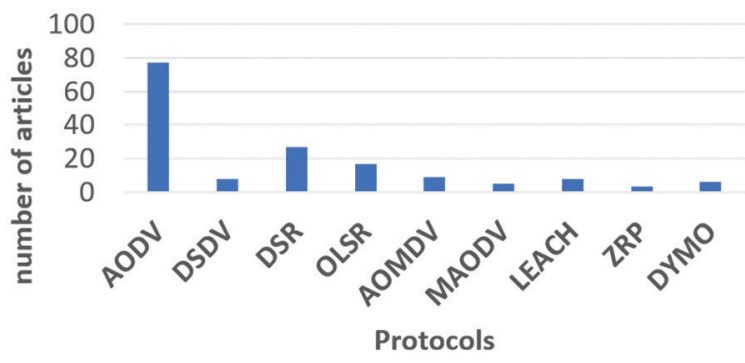

Fig. 5. Routing protocol choice by authors for the performance comparison

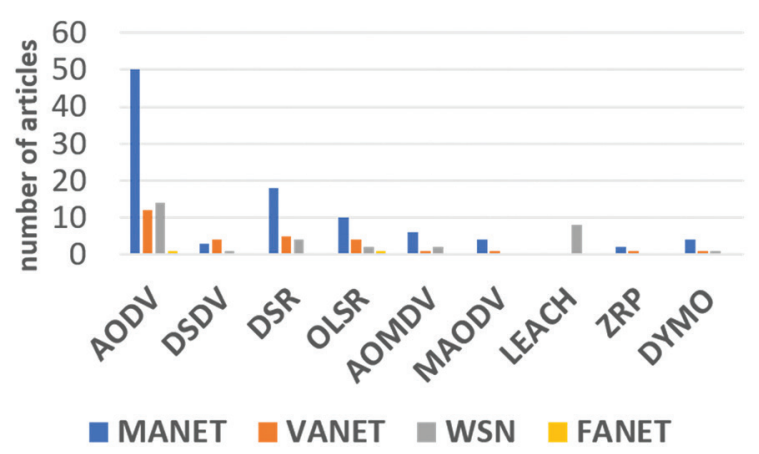

Fig. 6. Routing protocols usage based on MANET,VANET, WSN, FANET

Table 3. Protocols that have a low percentage of utilization

\begin{tabular}{|cccccc|}
\hline Ref & Protocol & Ref & Protocol & Ref & Protocol \\
\hline$[39]$ & CR-EAOMDV & {$[40]$} & LEACH-M & {$[41,42]$} & STFDR \\
\hline$[43]$ & EPC-AODV & {$[44]$} & LEACH-C & {$[45]$} & NCLR \\
\hline$[39]$ & AODMV-MR & {$[46]$} & EACHP & {$[47]$} & RIP \\
\hline$[39]$ & CAODV & {$[40]$} & EPCR & {$[48]$} & LAR \\
\hline$[49]$ & DAODV & {$[50]$} & FDCRP & {$[51]$} & WECRR \\
\hline$[52]$ & PMT-AODV & {$[51]$} & DFCR & {$[53]$} & A-CAR \\
\hline$[54]$ & FTDSR & {$[53]$} & IVD-CAGR & {$[55]$} & EAR \\
\hline$[56]$ & QoS-UMDSR & {$[53]$} & CSR & {$[20,57]$} & GSR \\
\hline$[56]$ & QOS-UDSR & {$[55]$} & CLB & & \\
\hline$[20]$ & SC-OLSR & {$[55]$} & DGLB & & \\
\hline
\end{tabular}

\subsection{MOBILITY MODELS}

Mobility models represent how the mobile nodes move inside the mobile ad-hoc network based on a specific pattern, position, and speed changes. Change in speed, position and pattern will result in the dis- placement of the mobile nodes in a particular region. In our review, different mobility models were selected by authors. Figure 7 shows that RWPM was the most used mobility model with 64 (37.9\%), SUMO/MOVE 13 (7.7\%), MOBISIM 5 (3\%), and 2 (1.2\%) for GMM and RWM, KRAUSS, RPGM, Bezier curves Mobility (BCM), Semi-random circular movement (SCRM). SUMO/ MOVE was used to create the movement of the vehicle in VANET. VANET can also use Vanet Mobisim for mobility models such as IDM (Intelligent Driver Model), IDMLC(Intelligent Driver Model with lane changes), IDM-IM (intelligent driver model with intersection management), and FTM (fluid traffic model) [22]. We have observed that RWPM was the most used mobility model in our review. SUMO/MOVE was most used to create the movement of the vehicle in VANET.

The use of one mobility model is useful to show the node's movement in a particular scenario for performance analysis, but which mobility model is the right one to choose for the performance analysis?. We can observe that an inclination is toward using only RWPM but using it alone will not guarantee a good result; it can give unreliable results, as shown in [23]. Comparing our result with the work in [24], RWPM is still the researcher's preferred mobility model. Another observation is that $49.7 \%$ of the paper did not mention the mobility models used. No mentioning the mobility model will lead to a work that cannot be repeatable or compared with others' works.

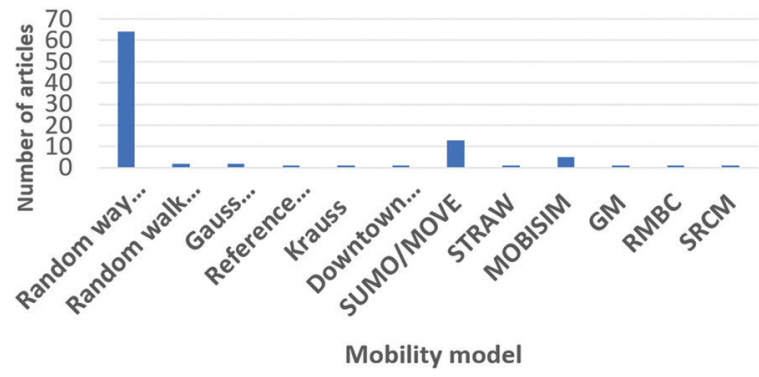

Fig. 7. Mobility model used by authors

\subsection{METRICS}

The metrics are essential for performance analysis. It gives you a big picture of the network performance in different ways. The choice of it is crucial, and our review shows in Figure 8 that 88 (52.1\%) out of 169 articles used Packet Delivery Ratio, 82 (48.5\%) End to End delay, 62 (36.7\%) Throughput, 45 (26.6\%) Overhead,36 (21.3\%) Energy consumption, 21 (12.4\%) Packet loss and Network Lifetime, 13 (7.7\%) Network lifetime, 7 (4.1\%) on Jitter and 5 (3\%) Latency. It can be observed that PDR, E2ED, Throughput, Overhead was the most chosen metric in our review. These metrics give the network's general performance, but adding other specific metrics can give the researcher more details for a particular aspect of the network's performance. Figure 9 shows the metric choice base on the field of study. In MANET 57 out of 90 (63.3\%) articles used PDR, 55 (61.1\%) E2ED, 41 (45.5\%) 
Throughput, 31 (34.4\%) Overhead, 17 (18.8\%) Total Energy consumption and Packet Loss, 6 (6.6\%). In VANET, 19 out of 32 (59.3\%) articles used PDR, 14 (43.7\%) E2ED, $8(25 \%)$ Throughput, and Overhead. In WSN, 11 out of 37 (29.7\%) articles used PDR, 13 (35.1\%) E2ED, 12 (32.4\%) Throughput, 5 (13.5\%) Overhead, 18 (48.6\%) Total energy consumption, and 12 (32.4\%) Network Lifetime. Form these results we can observe that Energy consumption was most used in WSN and MANET. Other choices of metrics that were less used can be found in Table 4.

In conclusion, challenges are there in terms of what metrics to choose for the performance analysis, as the trend is on using the old metrics (PDR, E2ED, Throughput, Overhead). Our comment is to use for a general view of the performance PDR, E2ED, Throughput, Overhead metrics. For detailed information on a particular aspect of a protocol's performance analysis, specific metrics can be used.

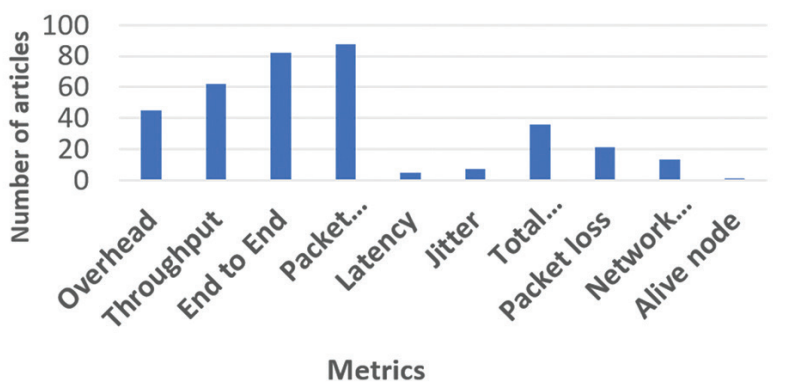

Fig. 8. Metric used by authors

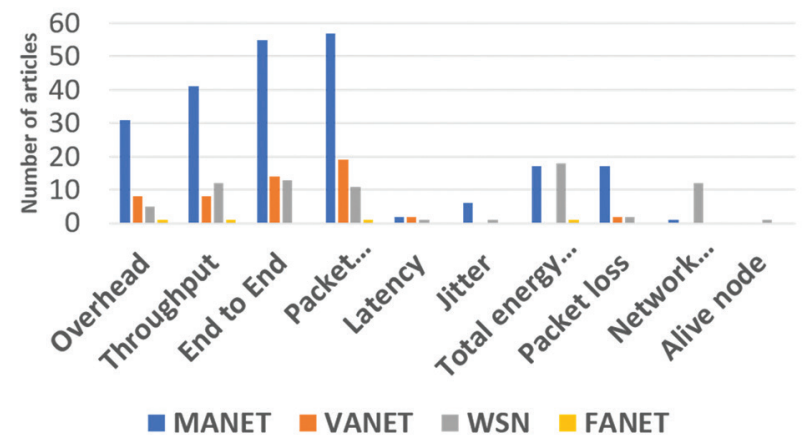

Fig. 9. Metric choice base on based on MANET,VANET, WSN, FANET

\section{B. What are the lessons learned and best practices in the performance analysis of ad hoc networks?}

In section 5, we have identified some challenges the researcher faces in choosing a simulator tool, a protocol to compare with or enhance, metrics, and the mobility model. The performance analysis of a protocol depends on these four parameters, which are essential for improving the result and future research in the ad hoc network. We have seen a significant improvement in ad hoc networks over the five years. New protocols have been created, enhancement of existing ones, and performance comparison with the existing ones. New metrics were created and apply in different scenarios with different mobility models. Nevertheless, there is room for improvement.

\section{Best practices}

Here we propose the best practices the researcher can use as a guide in terms of the simulator's choice, the protocol for comparison, mobility model, and metrics. The guide will help researchers and designers of software to improve the research quality in the Ad Hoc Networks.

- We recommended NS2 due to the availability of multiple models. Many protocols are implemented in it. The source code is available for free; the documentation and example can be found on the NS website and other pages (big user group) even though it has not been supported since 2010. Apart from NS2, which is our first choice, we also recommend NS3, MATLAB, and OMNET++ because these tools are activity updates and well documented.

- For selecting a specific protocol to compare with or to use for the enhancement, we recommend using the protocol that is closer in all aspects, e.g., security protocol with a security protocol. For example, the protocol should be recent, four to five years ago, for a better comparison. The same criteria should also be followed for the enhancement of a particular protocol.

- A selection of one mobility model can be accepted but not encourageable because it will not give the node movement's overall result. We encourage to use 2 or 4 mobility models for an excellent study of the performance analyses of a protocol. The best should be to use more mobility models to help other researchers see the protocol's strengths and weaknesses in different mobility models used.

- For a general view of a specific protocol's performance, we recommend using PDR, E2ED, Overhead, and Throughput, but for an in-depth analysis, the use of other metrics will be the right choice. Our recommendation is to add to the general metrics mention earlier one or two metrics; the best choice should be to use only specific metrics.

\section{Recommendation}

Here is our recommendation to the developer of simulator tools and the research community in ad hoc networks

Shared source code: The availability of code is essential for enhancing future research in ad hoc networks, but most published articles do not contain the source code and not even a link to a shared open free source code website. It is difficult for the researcher to compare the new protocol with the existing one or enhance it. We recommend the publisher to ask the author who voluntarily wants to share their source code with other researchers. 
Table 4. Other selective metrics used

\begin{tabular}{|c|c|c|c|c|c|}
\hline Ref & Metric & Ref & Metric & Ref & Metric \\
\hline [46] & Received message & {$[58,59]$} & Route discovery & {$[60]$} & Average SIP \\
\hline [46] & Alive sensor & {$[61]$} & Distributed key generation & {$[62]$} & Average travel time \\
\hline$[9,63-65]$ & Accuracy & {$[59,65]$} & Reachability & {$[66]$} & Decoded frame rate \\
\hline$[67]$ & Hello message & {$[68]$} & Data dissemination & [69] & Link expiration time \\
\hline$[70-72]$ & Bandwidth & {$[68]$} & Frequency of event & [53] & Broadcast problem \\
\hline [73] & Variance & {$[74]$} & Average number of route broken & {$[75]$} & Connectivity rate \\
\hline [73] & Traffic load & {$[76]$} & Wormhole Detection Ratio & [77] & Node trust value \\
\hline [3] & Average node weight & [9] & Location accuracy & {$[78]$} & FIFO packets dropped \\
\hline [3] & Number of accusations & [54] & Selfish nodes & {$[17,79,80]$} & Route failure \\
\hline
\end{tabular}

Benchmarks and acceptable scenario parameter: The result's creditability should be compared to a benchmark to prove that the result is acceptable, but Ad-Hoc Networks does not have a benchmark. The majority of authors always compare their results with other author's results. Parameters like the number of nodes, topology size, speed, and packet size are chosen arbitrarily by the researcher because there are no standard parameters acceptable to use. The lack of standard parameters can make the result doubtful. We recommend to the community to work on making standard benchmarks and scenarios parameter for ad hoc networks.

Simulation tools and Documentation: Simulation tools should contain more examples for new users to adapt to them. In our observation, the lack of more examples makes it difficult for the researcher to choose new simulation tools. New researchers preferred to use simulation tools that have more users so that it is very easy to find existing code and get advice from others researcher. New simulation tools are the most favourable tools the researcher must use because they have new features, but it is time-consuming for new researchers to be familiarized with them. A well-documented simulation tool with more examples and has helpful community can facilitate the use of new tools

3. Limitation of the study

This review used a selected journal in some digital libraries to analyze the performance of ad hoc networks. We used a limited search string to retrieve the articles in those selected journals, and articles published before 2015 were not included in the survey.

\section{CONCLUSION}

The performance analysis depends on several parameters included in the simulation tools; those parameters will influence the result if not well chosen. This survey analyzes articles based on these parameters; protocol, mobility models, metrics, and simulation tools. The statistic shows an inclination to used Ad-hoc On-Demand Distance Vector routing (AODV) for performance comparison and the researcher's enhancement. Network simulation 2 (NS2) was the most selected tool, but we observe a decline in its utilization in recent years. Random Waypoint Mobility model (RWPM) was the most used mobility model. We have found a high percentage of the published article did not mention the mobility models use; this will make the result difficult for performance comparison with other works. Packet Delivery Ratio (PDR), End to End Delay (E2ED) were the most used metrics. The survey explains some lessons learned in the study and proposes best practices and recommendations to the researcher and Ah Hoc Community.

\section{REFERENCES}

[1] M. R. Belgaum, S. Musa, M. MohdSu'ud, M. Alam, S. Soomro, Z. Alansari, "Secured Approach towards Reactive Routing Protocols Using Triple Factor in Mobile Ad Hoc Networks", Annals of Emerging Technologies in Computing, Vol. 3, 2019, pp. 32-40.

[2] M. R. Belgaum, S. Soomro, Z. Alansari, M. Alam, "Ideal Node Enquiry Search Algorithm (INESH) in MANETS", in Annals of Emerging Technologies in Computing Vol. 1, 2017, pp. 26-33.

[3] M. Masdari, M. Bidaki, F. Naghiloo, "Comprehensive Evaluation of the Localized Certificate Revocation in Mobile Ad Hoc Network", Wireless Personal Communications, Vol. 94, 2017, pp. 977-1001.

[4] G. M. Borkar, A. R. Mahajan, "A secure and trust based on-demand multipath routing scheme for self-organized mobile ad-hoc networks", Wireless Networks, Vol. 23, 2017, pp. 2455-2472.

[5] M. P. Arthur and K. Kannan, "Cross-layer based multiclass intrusion detection system for secure multicast communication of MANET in military networks", Wireless Networks, Vol. 22, 2016, pp. 1035-1059.

[6] N. Abbani, H. Artail, "Protecting data flow anonymity in mobile ad hoc networks that employ cooperative caching", Ad Hoc Networks, Vol. 26, 2015, pp. 69-87. 
[7] S. Tan, X. Li, Q. Dong, "Trust based routing mechanism for securing OSLR-based MANET", Ad Hoc Networks, Vol. 30, 2015, pp. 84-98.

[8] P. Kaur, D. Kaur, R. Mahajan, "Simulation based comparative study of routing protocols under wormhole attack in manet", Wireless Personal Communications, Vol. 96, No. 1, 2017, pp. 47-63.

[9] T. Karthikeyan, V. Brindha, P. Manimegalai, "Investigation on Maximizing Packet Delivery Rate in WSN Using Cluster Approach", Wireless Personal Communications, Vol. 103, 2018, pp. 3025-3039.

[10] T. Issariyakul, E. Hossain, "Introduction to network simulator 2 (NS2)", in Introduction to network simulator NS2, Springer, 2009, pp. 1-18.

[11] G. F. Riley, T. R. Henderson, "The ns-3 network simulator", in Modeling and tools for network simulation, Springer, 2010, pp. 15-34.

[12] A. Varga, R. Hornig, "An overview of the OMNeT++ simulation environment", Proceedings of the $1 \mathrm{st}$ international conference on Simulation tools and techniques for communications, networks and systems \& workshops, Marseille, France, 3-7 March 2008, pp. 1-10.

[13] Z. Lu, H. Yang, Unlocking the power of OPNET modeler. Cambridge University Press, 2012.

[14] X. Zeng, R. Bagrodia, M. Gerla, "GloMoSim: a library for parallel simulation of large-scale wireless networks", Proceedings. 12th Workshop on Parallel and Distributed Simulation, Banff, AB, Canada, 29-29 May 1998, pp. 154-161.

[15] B. Hahn, D. Valentine, Essential MATLAB for engineers and scientists. Academic Press, 2016.

[16] A. Benzerbadj, B. Kechar, A. Bounceur, B. Pottier, "Cross-Layer Greedy position-based routing for multihop wireless sensor networks in a real environment", Ad Hoc Networks, Vol. 71, 2018, pp. 135146.

[17] G. Singal, V. Laxmi, M. S. Gaur, V. Rao, "Moralism: mobility prediction with link stability based multicast routing protocol in MANETs", Wireless Networks, Vol. 23, 2017, pp. 663-679.

[18] F. Bai, A. Helmy, "A survey of mobility models", Wireless Adhoc Networks, Vol. 206, 2004, p. 147.
[19] M. Oche, A. B. Tambuwal, C. Chemebe, R. M. Noor, S. Distefano, "VANETs QoS-based routing protocols based on multi-constrained ability to support ITS infotainment services", Wireless Networks, Vol. 26, No. 3, 2020, pp. 1685-1715.

[20] Y. Hernafi, M. B. Ahmed, M. Bouhorma, "ACO and PSO algorithms for developing a new communication model for VANET applications in smart cities", Wireless Personal Communications, Vol. 96, No. 2, 2017, pp. 2039-2075.

[21] T. Li, J. Ma, C. Sun, "SRDPV: secure route discovery and privacy-preserving verification in MANETs", Wireless Networks, Vol. 25, No. 4, 2019, pp. 17311747.

[22] I. Zaimi, Z. S. Houssaini, A. Boushaba, M. Oumsis, D. Aboutajdine, "An evaluation of routing protocols for vehicular ad-hoc network considering the video stream", Wireless Personal Communications, Vol. 98, No. 1, 2018, pp. 945-981.

[23] J. Yoon, M. Liu, B. Noble, "Random waypoint considered harmful", Proceedings of the 22nd Annual Joint Conference of the IEEE Computer and Communications Societies, San Francisco, CA, USA, 30 March-3 April 2003, pp. 1312-1321.

[24] S. Kurkowski, T. Camp, M. Colagrosso, "MANET simulation studies: the incredibles", ACM SIGMOBILE Mobile Computing and Communications Review, Vol. 9, No. 4, 2005, pp. 50-61.

[25] D. Hiranandani, K. Obraczka, J. Garcia-LunaAceves, "MANET protocol simulations considered harmful: the case for benchmarking", IEEE Wireless Communications, Vol. 20, No. 4, 2013, pp. 82-90.

[26] S. Naicken, B. Livingston, A. Basu, S. Rodhetbhai, I. Wakeman, D. Chalmers, "The state of peer-to-peer simulators and simulations", ACM SIGCOMM Computer Communication Review, Vol. 37, No. 2, 2007, pp. 95-98.

[27] S. Kurkowski, W. Navidi, T. Camp, "Constructing manet simulation scenarios that meet standards", Proceedings of the IEEE International Conference on Mobile Adhoc and Sensor Systems, Pisa, Italy, 8-11 October 2007, pp. 1-9.

[28] A. Munjal, T. Camp, W. C. Navidi, "Constructing rigorous MANET simulation scenarios with realistic 
mobility", Proceedings of the European Wireless Conference, Lucca, Italy, 12-15 April 2010, pp. $817-$ 824.

[29] T. R. Andel, A. Yasinsac, "On the credibility of manet simulations", Computer, Vol. 39, no. 7, 2006, pp. 48-54.

[30] I. Ahmad, U. Ashraf, A. Ghafoor, "A comparative QoS survey of mobile ad hoc network routing protocols", Journal of the Chinese institute of engineers, Vol. 39, No. 5, 2016, pp. 585-592.

[31] J. Sánchez-García, J. García-Campos, M. Arzamendia, D. G. Reina, S. Toral, D. Gregor, "A survey on unmanned aerial and aquatic vehicle multi-hop networks: Wireless communications, evaluation tools and applications", Computer Communications, Vol. 119, 2018, pp. 43-65.

[32] J. M. García-Campos, J. Sánchez-García, D. Reina, S. Toral, F. Barrero, "An evaluation methodology for reliable simulation based studies of routing protocols in VANETs", Simulation modelling practice and theory, Vol. 66, 2016, pp. 139-165.

[33] P. P. Garrido, M. P. Malumbres, C. T. Calafate, "ns-2 vs. OPNET: a comparative study of the IEEE 802.11 e technology on MANET environments", Proceedings of the 1st international conference on Simulation tools and techniques for communications, networks and systems \& workshops, Marseille, France, 3-7 March 2008: Citeseer, pp. 1-10.

[34] E. Schoch, M. Feiri, F. Kargl, M. Weber, "Simulation of ad hoc networks: ns-2 compared to jist/swans", Proceedings of the 1st international conference on Simulation tools and techniques for communications, networks and systems \& workshops, Marseille, France, 3-7 March 2008, pp. 1-8.

[35] F. Kargl, E. Schoch, "Simulation of MANETs: a qualitative comparison between JiST/SWANS and ns2 ", Proceedings of the 1st international workshop on System evaluation for mobile platforms, San Juan, Puerto Rico, 11 June 2007, pp. 41-46.

[36] E. Weingartner, H. Vom Lehn, K. Wehrle, "A performance comparison of recent network simulators", Proceedings of the IEEE International Conference on Communications, Dresden, Germany, 14-18 June 2009, pp. 1-5.
[37] L. Begg, W. Liu, K. Pawlikowski, S. Perera, H. Sirisena, "Survey of simulators of next generation networks for studying service availability and resilience", Technical report, University of Canterbury, 2006.

[38] T. M. T. Pham, T. T. Nguyen, D. S. Kim, "Geographical awareness hybrid routing protocol in Mobile Ad Hoc Networks", Wireless Networks, Vol. 23, 2017, pp. 1-13.

[39] Y. H. Xu, Y. Wu, J. Song, "Joint Channel Assignment and Routing Protocol for Cognitive Radio Wireless Sensor Networks", Wireless Personal Communications, Vol. 97, 2017, pp. 41-62.

[40] N. Ismat, R. Qureshi, S. Mumtaz ul Imam, "Adaptive Power Control Scheme for Mobile Wireless Sensor Networks", Wireless Personal Communications, Vol. 106, 2019, pp. 2195-2210.

[41] Y. H. Robinson, R. S. Krishnan, E. G. Julie, R. Kumar, L. H. Son, P. H. Thong, "Neighbor Knowledgebased Rebroadcast algorithm for minimizing the routing overhead in Mobile Ad-hoc Networks", Ad Hoc Networks, Vol. 93, 2019, p. 101896.

[42] J. Sathiamoorthy, B. Ramakrishnan, M. Usha, "STFDR: Architecture of Competent Protocol for Efficient Route Discovery and Reliable Transmission in CEAACK MANETs", Wireless Personal Communications, Vol. 97, 2017, pp. 5817-5839.

[43] S. Vidhya, T. Sasilatha, "Secure Data Transfer Using Multi Layer Security Protocol with Energy Power Consumption AODV in Wireless Sensor Networks", Wireless Personal Communications, Vol. 103, 2018, pp. 3055-3077.

[44] G. Dhand, S. S. Tyagi, "SMEER: Secure Multi-tier Energy Efficient Routing Protocol for Hierarchical Wireless Sensor Networks", Wireless Personal Communications, Vol. 105, 2019, pp. 17-35.

[45] N. Kaur, R. Singhai, "Analysis of Traffic Impact on Proposed Congestion Control Scheme in AODV", Wireless Personal Communications, Vol. 109, 2019, pp. 1395-1418.

[46] H. Barati, A. Movaghar, A. M. Rahmani, "EACHP: Energy Aware Clustering Hierarchy Protocol for Large Scale Wireless Sensor Networks", Wireless Personal Communications, Vol. 85, 2015, pp. 765-789. 
[47] V. K. Verma, S. Singh, N. P. Pathak, "Optimized Battery Models Observations for Static, Distance Vector and On-Demand Based Routing Protocols Over 802.11 Enabled Wireless Sensor Networks", Wireless Personal Communications, Vol. 81, 2015, pp. 503-517.

[48] K. K. Rana, S. Tripathi, R. S. Raw, "Analytical analysis of improved directional location added routing protocol for VANETS", Wireless Personal Communications, Vol. 98, 2018, pp. 2403-2426.

[49] M. Rajesh Babu, G. Usha, "A Novel Honeypot Based Detection and Isolation Approach (NHBADI) To Detect and Isolate Black Hole Attacks in MANET", Wireless Personal Communications, Vol. 90, 2016, pp. 831-845.

[50] S. Jayaraman, R. Bhagavathiperumal, U. Mohanakrishnan, "A Three Layered Peer-to-Peer Energy Efficient Protocol for Reliable and Secure Data Transmission in EAACK MANETs", Wireless Personal Communications, Vol. 102, 2018, pp. 201 227.

[51] K. Haseeb, K. A. Bakar, A. Ahmed, T. Darwish, I. Ahmed, "WECRR: Weighted Energy-Efficient Clustering with Robust Routing for Wireless Sensor Networks", Wireless Personal Communications, Vol. 97, 2017, pp. 695-721.

[52] X. Anita, M. A. Bhagyaveni, J. Martin Leo Manickam, "Collaborative Lightweight Trust Management Scheme for Wireless Sensor Networks", Wireless Personal Communications, Vol. 80, 2015, pp. 117-140.

[53] M. U. Hassan, M. H. Rehmani, Y. Faheem, "Performance evaluation of broadcasting strategies in cognitive radio networks", Wireless Networks, Vol. 25, 2019, pp. 999-1016.

[54] S. A. Thorat, P. J. Kulkarni, "Opportunistic Routing in Presence of Selfish Nodes for MANET", Wireless Personal Communications, Vol. 82, 2015, pp. 689708.

[55] T. M. Rajeh, A. I. Saleh, L. M. Labib, "A New Cooperative Balancing Routing (CBR) Protocol to Enhance the Lifetime of Wireless Sensor Networks", Wireless Personal Communications, Vol. 98, 2018, pp. 2623-2656.
[56] V. V. Mandhare, R. R. Manthalkar, V. R. Thool, "Novel Approach for Cache Update on Multipath DSR Protocol in MANET for QoS Support", Wireless Personal Communications, Vol. 98, 2018, pp. 505-519.

[57] S. K. Bhoi, P. M. Khilar, "Self soft fault detection based routing protocol for vehicular ad hoc network in city environment", Wireless Networks, Vol. 22, 2016, pp. 285-305.

[58] S. Tabatabaei, M. Teshnehlab, S. J. Mirabedini, "Fuzzy-Based Routing Protocol to Increase Throughput in Mobile Ad Hoc Networks", Wireless Personal Communications, Vol. 84, 2015, pp. $2307-$ 2325.

[59] S. S. Basurra, M. De Vos, J. Padget, Y. Ji, T. Lewis, S. Armour, "Energy efficient zone based routing protocol for MANETs", Ad Hoc Networks, Vol. 25, 2015, pp. 16-37.

[60] F. Alshahwan, M. Alshamrani, A. A. Amer, "Dynamic Novel Cross-Layer Performance Enhancement Approach for SIP over OLSR", IEEE Access, Vol. 6, 2018, pp. 71947-71964.

[61] H. Kojima, N. Yanai, J. P. Cruz, "ISDSR+: Improving the Security and Availability of Secure Routing Protocol", IEEE Access, Vol. 7, 2019, pp. 7484974868.

[62] J. S. Pan, I. S. Popa, C. Borcea, "DIVERT: A distributed vehicular traffic re-routing system for congestion avoidance", IEEE Transactions on Mobile Computing, Vol. 16, 2017, pp. 58-72.

[63] K. Raja, A. Deivasigamani, V. Ravi, "A Reliant Certificate Revocation of Malicious Nodes in MANETs", Wireless Personal Communications, Vol. 90, 2016, pp. 435-455.

[64] A. Pal, P. Dutta, A. Chakrabarti, J. P. Singh, S. Sadhu, "Biogeographic-Based Temporal Prediction of Link Stability in Mobile Ad Hoc Networks", Wireless Personal Communications, Vol. 104, 2019, pp. 217-233.

[65] A. E. Hilal, A. B. MacKenzie, "A distributed coalition game model for cooperation in MANETs", Ad Hoc Networks, Vol. 85, 2019, pp. 46-59.

[66] S. González, W. Castellanos, P. Guzmán, P. Arce, J. C. Guerri, "Simulation and experimental testbed for adaptive video streaming in ad hoc networks", Ad Hoc Networks, Vol. 52, 2016, pp. 89-105. 
[67] D. S. Sakkari, T. G. Basavaraju, "GCCT: A GraphBased Coverage and Connectivity Technique for Enhanced Quality of Service in WSN", Wireless Personal Communications, Vol. 85, 2015, pp. 1295 1315.

[68] M. Guerroumi, A. S. K. Pathan, "Hybrid data dissemination protocol (HDDP) for wireless sensor networks", Wireless Networks, Vol. 24, 2018, pp. 1739-1754.

[69] A. Naushad, G. Abbas, Z. H. Abbas, A. Pagourtzis, "Novel strategies for path stability estimation under topology change using Hello messaging in MANETs", Ad Hoc Networks, Vol. 87, 2019, pp. 7699.

[70] S. R. Malwe, N. Taneja, G. P. Biswas, "Enhancement of DSR and AODV Protocols Using Link Availability Prediction", Wireless Personal Communications, Vol. 97, 2017, pp. 4451-4466.

[71] M. Malathi, S. Jayashri, "Modified Bi-directional Routing with Best Afford Path (MBRBAP) for Routing Optimization in MANET", Wireless Personal Communications, Vol. 90, 2016, pp. 861-873.

[72] J. K. Jayabarathan, A. Sivanantharaja, S. Robinson, "Quality of Service Enhancement of Mobile Adhoc Networks Using Priority Aware Mechanism in AODV Protocol", Wireless Personal Communications, Vol. 96, 2017, pp. 5897-5909.

[73] Y. Qin, L. Li, X. Zhong, Y. Yang, C. L. Gwee, "A CrossLayer QoS Design with Energy and Traffic Balance Aware for Different Types of Traffic in MANETs", Wireless Personal Communications, Vol. 85, 2015, pp. 1429-1449.
[74] C. Lal, V. Laxmi, M. S. Gaur, M. Conti, "Enhancing QoE for video streaming in MANETs via multi-constraint routing", Wireless Networks, Vol. 24, 2018, pp. 235-256.

[75] W. Wang, J. Wang, M. Wang, B. Wang, W. Zhang, "A realistic mobility model with irregular obstacle constraints for mobile ad hoc networks", Wireless Networks, Vol. 25, 2019, pp. 487-506.

[76] T. T. Vo, N. T. Luong, D. Hoang, "MLAMAN: a novel multi-level authentication model and protocol for preventing wormhole attack in mobile ad hoc network", Wireless Networks, Vol. 0123456789, 2018.

[77] A. M. Shabut, K. P. Dahal, S. K. Bista, I. U. Awan, "Recommendation Based Trust Model with an Effective Defence Scheme for MANETs", IEEE Transactions on Mobile Computing, Vol. 14, 2015, pp. 2101-2115.

[78] W. A. Jabbar, W. K. Saad, M. Ismail, "MEQSA-OLSRv2: A multicriteria-based hybrid multipath protocol for energy-efficient and QoS-aware data routing in MANET-WSN convergence scenarios of IoT", IEEE Access, Vol. 6, 2018, pp. 76546-76572.

[79] O. S. Gnana Prakasi, P. Varalakshmi, "Decision Tree Based Routing Protocol (DTRP) for Reliable Path in MANET", Wireless Personal Communications, Vol. 109,2019 , pp. 257-270.

[80] L. Sayad, L. Bouallouche-Medjkoune, D. Aissani, "IWDRP: An Intelligent Water Drops Inspired Routing Protocol for Mobile Ad Hoc Networks", Wireless Personal Communications, Vol. 94, 2017,pp. 2561-2581. 